\title{
Treatment Couch Modeling in the Treatment Planning System Eclipse
}

\author{
Daniela Wagner* and Hilke Vorwerk \\ Department of Radiotherapy and Radiooncology, University Hospital Goettingen, Robert-Koch-Str. 40, 37075 Goettingen, Germany
}

\begin{abstract}
Purpose: Previously in treatment planning systems (TPS) the treatment couch was expected to be made out of air-equivalent material due to the used material (Carbon). Some studies have already shown that the treatment couch cannot be neglected during treatment planning. Nowadays the manufacturer of TPS implemented the feasibility to insert treatment couch structures. This study aimed to find the correct modeling of the treatment couch parameters in the TPS Eclipse.

Method: The Varian Exact Treatment Couch consists of a carbon board (length $2.5 \mathrm{~cm}$ ) and two moveable rails (length $8.5 \mathrm{~cm}$ ) underneath. The treatment couch can be modeled in TPS by changing the Hounsfield units (HU) for each part of the treatment couch. For low and high photon energies the attenuation of the treatment couch was measured at a Clinac $2300 \mathrm{C} / \mathrm{D}$ and in the TPS the attenuation of the treatment couch model was determined for different sets of HU values. Measured and calculated attenuations were compared to each other.

Results: Minimum aberration between the calculated and measured attenuation of treatment couch were found for the $\mathrm{HU}$ values of $-750 \mathrm{HU}$ for the carbon plate, $-995 \mathrm{HU}$ for the filling of the carbon plate and $225 \mathrm{HU}$ for the rails. Additionally it was found that the attenuation is dependent on the gantry angle. Like expected the highest attenuation was found in the region of the rails underneath the treatment couch.

Conclusion: For Varian Exact Treatment Couch the HU values should be adjusted to $-750 \mathrm{HU}$ for the carbon plate, $-995 \mathrm{HU}$ for the filling of the carbon plate and $225 \mathrm{HU}$ for the rails. The same $\mathrm{HU}$ set can be used for low and high photon energies. With the correct set of HU values the treatment couch is modeled correctly in the TPS Eclipse.
\end{abstract}

Keywords: Radiation therapy; Treatment planning; Couch structures; Attenuation

\section{Introduction}

The treatment couch and some of the positioning devices of patients receiving radiotherapy treatment are made of a material (carbon) which has low attenuation properties for the photon energies used in the radiotherapy. Previous investigations showed the suitability of carbon fiber tabletops in radiotherapy and their very low attenuation properties for a range of X-ray energies and field sizes (De Ost et al., 1997; Higgins et al., 2001; Meara and Langmack, 1998). The Carbon treatment couch is designed to minimize the potential blocking of the radiation treatment beam for conventional treatment techniques (Buckle, 2005; Meyer et al., 2001; Muthuswamy, 1999; Spezi et al., 2008). Some studies have shown that the treatment couch is not negligible during treatment planning process whereas the dose delivery is performed with treatment couch (Myint et al., 2006; Poppe et al., 2007; Spezi and Ferri, 2007; Vieira et al., 2003).

The effect of the treatment couch to the dose distribution depends on the gantry angle. The effect is larger for posterior beam angles (McCormack et al., 2005). The attenuation increased from $2 \%$ for beam angles as the gantry approaches the plane to $9 \%$ for posterior beam angles. This could have serious implications regarding dose to the treatment volume for treatments requiring posterior oblique angles of incidence with a possible correction factor necessary in monitor unit calculations (McCormack et al., 2005). Side bars or other design features specific of each carbon fiber tabletop disturbed the dose distribution at the isocenter at specific gantry angles (Gillis et al., 2005; Munjal et al., 2006).

It has also to be taken into account that the skin dose increased due to the carbon couch top. The treatment couch operates as a buildup (Meara and Langmack, 1998). The carbon fiber tabletop significantly decreases the skin-sparing effect. The dosimetric effect of the tabletop may be higher, especially for the intensity-modulated radiation therapy depending on the beam orientation (Meydanci and Kemikler, 2008). The potential for dose delivery errors or increased whole-body dose should be carefully considered by newest delivery technologies (Galvin et al., 2004).

Due to some studies recommending the consideration of the treatment couch during the treatment planning process investigations are made to construct the treatment couch in the treatment planning system. The appropriate contouring together with relevant density information for the contours is sufficient for adequate modeling of carbon fiber supporting devices by modern commercial treatment planning systems and can reduce the differences between the dose calculated without consideration of the treatment couch and the dose calculated with consideration of the constructed treatment couch (Mihaylov et al., 2008).

Using the treatment planning system Eclipse Version 8.9 from Varian Medical Systems (Helsinki, Finland) in combination with the Anisotropic Analytical Algorithm (AAA) the treatment couch

*Corresponding author: Daniela Wagner, Department of Radiotherapy and Radiooncology, University Hospital Goettingen, Robert-Koch-Str. 40, 37075 Goettingen, Germany, Tel: +49-551-398866; Fax: +49-551-396192; E-mail: d.m.wagner@med.uni-goettingen.de

Received September 28, 2010; Accepted October 18, 2010; Published October 18,2010

Citation: Wagner D, Vorwerk H (2011) Treatment Couch Modeling in the Treatment Planning System Eclipse. J Cancer Sci Ther 3: 007-012. doi:10.4172/19485956.1000049

Copyright: @ 2011 Wagner D, et al. This is an open-access article distributed under the terms of the Creative Commons Attribution License, which permits unrestricted use, distribution, and reproduction in any medium, provided the original author and source are credited. 
structures can be easily inserted. The AAA is a 3D pencil Beam convolution/superposition algorithm that uses separate Monte Carlo derived modeling for primary photons, scattered extra-focal photons and electrons scattered from the beam limiting devices (Ulmer et al., 1995; Ulmer et al., 2005). The treatment couch can be considered in optimization process and dose distribution calculation. The aim of this study was to find the correct modeling parameters of the treatment couch (Varian Exact Couch) in the treatment planning system Eclipse. Therefore measurements at Varian Clinac $2300 \mathrm{C} / \mathrm{D}$ and calculation in treatment planning system Eclipse were made to determine the attenuation of the treatment couch for low and high photon energies. Both attenuations were compared to each other and the modeling of treatment couch was adapted till measured and calculated dose fitted together.

\section{Methods and Materials}

\section{Setup for measurements}

For the measurements of attenuation of the treatment couch a cylindrical phantom made of PMMA (Polymethylmethacrylat PMMA) was set up on the treatment couch. The reading of an ionization chamber with an active measuring volume of $0.3 \mathrm{~cm}^{3}$ (Farmer chamber, PTW, Freiburg, Germany) was determined for the gantry angles between $0^{\circ}$ and $360^{\circ}$ (every $5^{\circ}$ ). The measurements were carried out for both photon energies, $6 \mathrm{MV}_{\text {photons }}$ and $20 \mathrm{MV}_{\text {photons }}$, with a field size of $10 \mathrm{~cm} \times 10 \mathrm{~cm}$. The ionization chamber was at source to detector distance (SDD) of $100 \mathrm{~cm}$ and cylindrical surrounded by 10 $\mathrm{cm}$ PMMA. The reading of ionization chamber was determined three times for middle position and external position of rails underneath the treatment couch (Figure 1). 100 monitor units (MU) were delivered.

\section{Treatment couch}

The Varian Exact Treatment Couch consists of a carbon plate with a thickness of $2.5 \mathrm{~cm}$ and two carbon rails beneath the carbon plate for stabilization purposes with a height of $8.5 \mathrm{~cm}$. The couch top is made of carbon shell with a length of $1.0 \mathrm{~mm}$ at the top and of 2.5 $\mathrm{mm}$ at the bottom filled with foam. The rails are made of a shell with a length of about $10 \mathrm{~mm}$ filled with air. The moveable rails can be moved in every position underneath the treatment couch. There are no fixed positions for the reproducibility of the position of the rails. In practice the positions in the middle and outer most are used.

\section{Calculation with TPS eclipse}

In TPS Eclipse version 8.9 from Varian Medical System the treatment couch from the same manufacturer can be inserted in every CT-dataset ( $C T=$ computertomogaphy) of a patient or phantom. The HU values of each part of treatment couch can be typed in. With these $\mathrm{HU}$ values the attenuation of treatment couch is assessed. For the treatment couch the manufacturer set $-300 \mathrm{HU}$ for the Carbon plate, $-1000 \mathrm{HU}$ for the foam inside the Carbon plate and $200 \mathrm{HU}$ for the rails as default. To determine the correct $\mathrm{HU}$ values of each

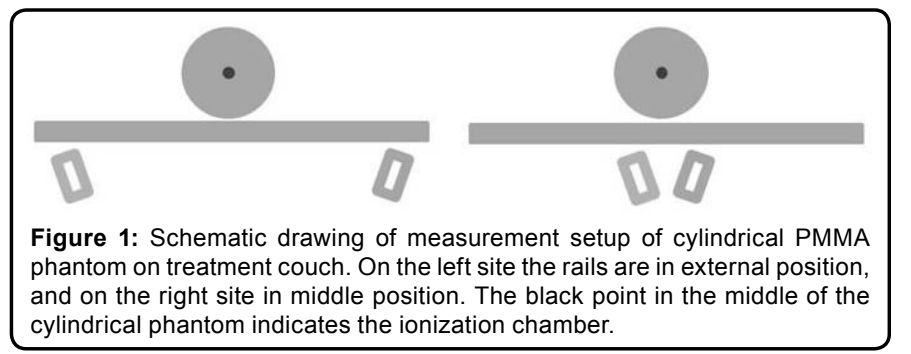

part of treatment couch the attenuation of the treatment couch at a Clinac $2300 \mathrm{C} / \mathrm{D}$ was determined. The same setup of cylindrical phantom on treatment couch was used in TPS and the attenuation of the treatment couch in TPS was determined. Therefore a photon field with a field size of $10 \mathrm{~cm} \times 10 \mathrm{~cm}$ for both photon energies, $6 \mathrm{MV}_{\text {photons }}$ and $20 \mathrm{MV}_{\text {photons }}$, was set up on the CT-scan of the PMMA phantom with ionization chamber inserted and the mean dose to the active volume of the ionization chamber was determined for the gantry angles between $0^{\circ}$ and $360^{\circ}$ (every $5^{\circ}$ ). The isocenter of the photon field was set at the middle of the cylindrical phantom. The dose distribution was calculated with the anisotropic analytical algorithm AAA with a grid size of $2 \mathrm{~mm}$ x $2 \mathrm{~mm}$ x $2 \mathrm{~mm}$. The treatment plan was normalized to $100 \mathrm{MU}$.

\section{Calculation of attenuation of treatment couch}

The attenuation of treatment coach was calculated as ratio of measured value with treatment couch $M_{\text {couch }}$ to measured value without treatment coach $\mathrm{M}_{\text {no couch }}$ :

$$
\text { attenuation }=\frac{\mathrm{M}_{\text {couch }}}{\mathrm{M}_{\text {no couch }}} .100 \%
$$

For the measurements in TPS the measured values with and without treatment couch could be determined for all gantry angles. For the measurement at the Clinac $2300 \mathrm{C} / \mathrm{D}$ the cylindrical phantom was setup on treatment couch. To calculate the attenuation of treatment couch the measured value of $0^{\circ}$ was used for the measured value without treatment couch due to the cylindrical setup.

\section{Analysis}

Both, the attenuation calculated in TPS (calculated attenuation) and calculated with the measurements at Clinac $2300 \mathrm{C} / \mathrm{D}$ (measured attenuation) were compared to each other to find the minimum aberration. For the set of HU values with minimum aberration the best modeling of treatment couch in TPS is given.

\section{Uncertainty budget}

Since the ratio of measured attenuation to calculated attenuation was considered as the end result, the following sources contributing to the overall uncertainty of the result were identified:

Ionization chamber measurement method

Dose calculation of the treatment planning system

Positioning of the cylindrical phantom on treatment couch

In our study, the uncertainty components for ionization chamber measurement method had to be taken into account due to positioning of cylindrical phantom using the isocentric laser system in treatment room, the response of ionization chamber reading and an additional component for directionally response of ionization chamber by rotation of the chamber around its axis by more than $10^{\circ}$. As a result, an uncertainty of the measured values with the Clinac $2300 \mathrm{C} / \mathrm{D}$ of $2.2 \%$ was obtained. The components of the combined uncertainty for the measured values with TPS are shown in Table 1.

For the accuracy of the dose calculation, the manufacturer specifies $1.0 \%$ for unblocked photon fields. The uncertainty of the treatment machine's basic data measurements had to be taken into consideration within $2 \mathrm{~mm}$. The consideration of $2 \mathrm{~mm}$ contained the exact positioning of the ionization chamber during basic data measurements for the TPS before clinical operation. However, the combined relative standard uncertainty of the measured values with TPS was only $1.0 \%$. The different contributions are listed in Table 1. All uncertainties are stated as $\mathrm{k}=1(1 \mathrm{r})$ standard uncertainties. 


\begin{tabular}{|l|l|l|l|}
\hline \multicolumn{2}{|l|}{ Calculated values } & \multicolumn{2}{l|}{ Measured values } \\
\hline Components for calculated values & Relative standard uncertainties & Components for measured values & Relative standard uncertainties \\
\hline Dose calculation of the treatment planning system & $1.0 \%$ & Positioning of cylindrical PMMA phantom & $0.3 \%$ \\
\hline Basic data measurements & $0.3 \%$ & Response of ionization chamber & $2.0 \%$ \\
\hline & & $\begin{array}{l}\text { directionally response of ionization } \\
\text { chamber by rotation of the chamber } \\
\text { around its axis by more than } 10^{\circ}\end{array}$ & $0.1 \%$ \\
\hline Total & $\mathbf{1 . 0 \%}$ & $\mathbf{2 . 2 \%}$ & \\
\hline
\end{tabular}

Table 1: Uncertainty budget.

\begin{tabular}{|l|l|l|l|l|l|l|}
\hline Treatment couch parts & Set Default & Set 1 & Set 2 & Set 3 & Set 4 \\
\hline Carbon plate & $-300 \mathrm{HU}$ & $-280 \mathrm{HU}$ & $-700 \mathrm{HU}$ & $-700 \mathrm{HU}$ & $-700 \mathrm{HU}$ \\
\hline Filling & $-1000 \mathrm{HU}$ & $-800 \mathrm{HU}$ & $-960 \mathrm{HU}$ & $-960 \mathrm{HU}$ & $-960 \mathrm{HU}$ & $-960 \mathrm{HU}$ \\
\hline Rails & $200 \mathrm{HU}$ & $200 \mathrm{HU}$ & $100 \mathrm{HU}$ & $200 \mathrm{HU}$ & $-750 \mathrm{HU}$ \\
\hline
\end{tabular}

Table 2: $\mathrm{HU}$ values for the different parts of treatment couch. The Varian exact treatment couch consists of a Carbon plate with a filling and of two rails underneath the Carbon plate for stabilization purposes.

\begin{tabular}{|c|c|c|c|c|}
\hline \multicolumn{5}{|c|}{ Middle position of rails } \\
\hline & \multicolumn{2}{|l|}{$6 \mathrm{MV}_{\text {photons }}$} & \multicolumn{2}{|l|}{$20 \mathrm{MV}_{\text {photons }}$} \\
\hline & Carbon couch top & Rails & Carbon couch top & Rails \\
\hline Set Default & much too high & good & much too high & good \\
\hline Set 1 & much too high & good & much too high & good \\
\hline Set 2 & too high & too low & too low & good \\
\hline Set 3 & too high & good & good & good \\
\hline Set 4 & too high & good & too low & too high \\
\hline Set 5 & too low & too low & good & good \\
\hline Set 6 & good & good & good & good \\
\hline \multicolumn{5}{|c|}{ External position of rails } \\
\hline & \multicolumn{2}{|l|}{$6 \mathrm{MV}_{\text {photons }}$} & \multicolumn{2}{|l|}{$20 \mathrm{MV}_{\text {photons }}$} \\
\hline & Carbon couch top & Rails & Carbon couch top & Rails \\
\hline Set Default & too high & good & much too high & good \\
\hline Set 1 & too high & good & much too high & good \\
\hline Set 2 & good & too low & too high & good \\
\hline Set 3 & good & good & good & good \\
\hline Set 4 & good & too high & good & good \\
\hline Set 5 & too low & too low & too low & too low \\
\hline Set 6 & good & good & good & good \\
\hline
\end{tabular}

Table 3: Results of the comparison between measured and calculated attenuation for the subsumed gantry angles. Aberration of <2.2\% indicates a good agreement between calculated and measured attenuation, a positive aberration $>2.2 \%$ too low $\mathrm{HU}$ values and a negative attenuation $>2.2 \%$ too high $\mathrm{HU}$ values.

\begin{tabular}{|l|l|l|}
\hline & Mean difference & Range \\
\hline Set Default & $0.84 \% \pm 0.15 \%$ & $-3.63 \%$ to $8.43 \%$ \\
\hline Set 1 & $1.08 \% \pm 0.15 \%$ & $-2.60 \%$ to $9.46 \%$ \\
\hline Set 2 & $0.35 \% \pm 0.32 \%$ & $-6.63 \%$ to $6.66 \%$ \\
\hline Set 3 & $0.21 \% \pm 0.17 \%$ & $-6.11 \%$ to $8.83 \%$ \\
\hline Set 4 & $0.25 \% \pm 0.24 \%$ & $-5.40 \%$ to $4.03 \%$ \\
\hline Set 5 & $0.57 \% \pm 0.40 \%$ & $6.16 \%$ to $5.72 \%$ \\
\hline Set 6 & $0.15 \% \pm 0.13 \%$ & $-2.02 \%$ to $1.96 \%$ \\
\hline
\end{tabular}

Table 4: Mean difference between measured and calculated attenuation of treatment couch, its standard uncertainty and its range. The mean difference was calculated for both photon energies and both positions of rails.

\section{Results}

To determine the attenuation measurements were made at a Clinac $2300 \mathrm{C} / \mathrm{D}$ and calculations were made in TPS Eclipse. The measured values were determined for rails in middle and external position, for both photon energies, $6 \mathrm{MV}_{\text {photons }}$ and $20 \mathrm{MV}_{\text {photons }}$ and for gantry angles from $0^{\circ}$ to $360^{\circ}$ (every $5^{\circ}$ ). Beginning with the default settings of $\mathrm{HU}$ values given by manufacturer the correct $\mathrm{HU}$ values were approached. In the end, seven HU-sets for Carbon plate and its filling and for rails were used till the optimal modeling of treatment couch was reached. The HU-sets are listed in Table 2.
For easy comparison of attenuation the measured values of the gantry angles were subsumed which were affected by the Carbon plate and by Carbon plate and rails. Gantry angles from $120^{\circ}$ to $150^{\circ}$ and from $210^{\circ}$ to $240^{\circ}$ were subsumed for Carbon plate attenuation, rails in middle position and gantry angles from $140^{\circ}$ to $220^{\circ}$, rails in external position, respectively. For Carbon plate and rails gantry angles from $120^{\circ}$ to $135^{\circ}$ and from $225^{\circ}$ to $240^{\circ}$ were subsumed, rails in middle position and gantry angles from $160^{\circ}$ to $170^{\circ}$ and from $190^{\circ}$ to $200^{\circ}$, rails in external position, respectively. In Table 3 the results of the comparison between measured and calculated attenuation for the subsumed gantry angles are listed. An aberration smaller than 
$2.2 \%$ (within measurement accuracy, Table 1) was defined as 'good agreement' for the HU values used, a positive aberration larger than $2.2 \%$ as 'too low' and negative aberrations larger than $2.2 \%$ as 'too high'. Much too high HU values for Carbon plate were found for Set Default and Set 1 (aberration $>50 \%$ to the measured values). For Set 2, Set 3 and Set 4 the aberration was between $2.2 \%$ and $5 \%$. For these sets the HU values were a little bit too high for Carbon plate. Aberration larger than 5\% was found for Set 5, except for photon energy of $20 \mathrm{MV}_{\text {photons }}$ and rails in middle position. For Set 5 the $\mathrm{HU}$ values were set too low for Carbon plate. A good agreement for all measurements was found for Set 6 and therefore a good modeling of treatment couch top in TPS. For Carbon plate and rails aberrations smaller $2.2 \%$ were found mainly for photon energy of $20 \mathrm{MV}$ For photon energy $6 \mathrm{MV}_{\text {photons }}$ the HU values were set too low for Set 2 and Set 5 . Set 4 showed too high HU values for photon energy 6 $\mathrm{MV}_{\text {photons }}$ and external position of the rails, as well as for $20 \mathrm{MV}_{\text {pl }}$ and middle position of the rails. Figure 2 shows the measured and calculated attenuation of treatment couch for both photon energies, for middle and external position of the rails. For Carbon plate the HU values were much too high for Set Default und Set 1, too low for Set 5, a little bit too high for Set 2, Set 3 and Set 4 and finally optimal for Set 6 . For Carbon plate and rails the HU values were too low for Set 2 and Set 5, too high for Set 4 and optimal for Set Default, Set 1, Set 3 and Set 6 (Figure 2).
In Table 4 the mean difference between measured and calculated attenuation is listed for both photon energies, for middle and external position of the rails. A minimum was found for Set 6 with $0.15 \%$ with its standard uncertainty of $0.13 \%$ and the range from $-2.02 \%$ to $1.96 \%$. The best agreement between measured and calculated attenuation was found for Set 6 (Figure 2 and Table 4). In Figure 2 it can also be seen that the attenuation increased in the region of the rails and is less in the region of the Carbon plate.

To verify the results a CT scan was made of Carbon plate and rails before installation. The $\mathrm{HU}$ values could be verified to $-750 \mathrm{HU}$ for Carbon plate, $-995 \mathrm{HU}$ for its filling and $225 \mathrm{HU}$ for rails.

\section{Discussion}

The aim of this study was to model the treatment couch in TPS by finding the optimal HU values for each part of treatment couch. By typing in the optimal $\mathrm{HU}$ values the attenuation of treatment couch is correctly considered during treatment planning.

The aberration and therefore the HU values between measured and calculated attenuations of Set Default and Set 1 were too high. Beginning with the study of Vanetti et al. [20], who found out HU values of $-700 \mathrm{HU}$ for Carbon plate and $-960 \mathrm{HU}$ for its filling for another type of treatment couch, $-700 \mathrm{HU}$ was used for Carbon plate

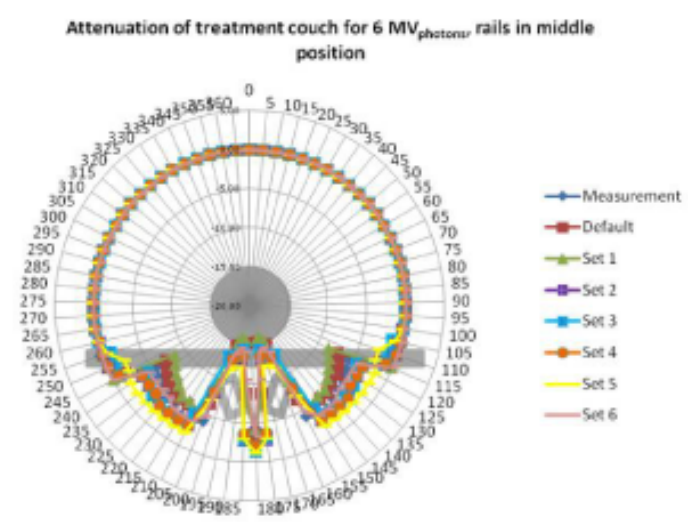

(a)

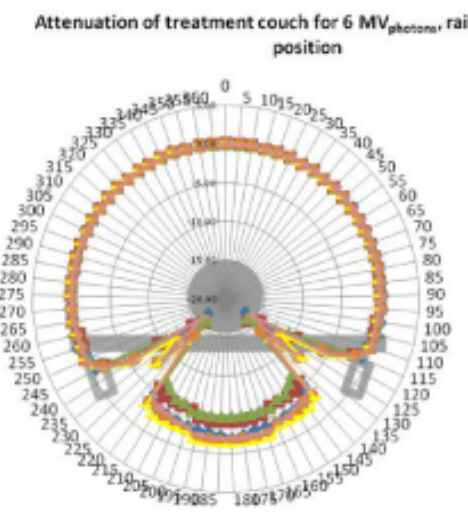

(c)

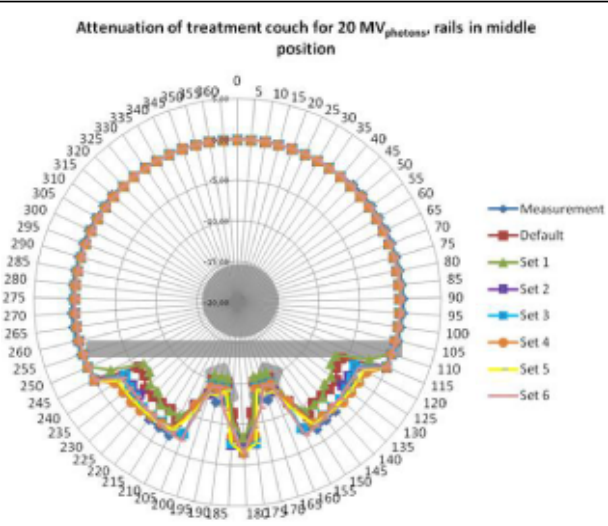

(b)

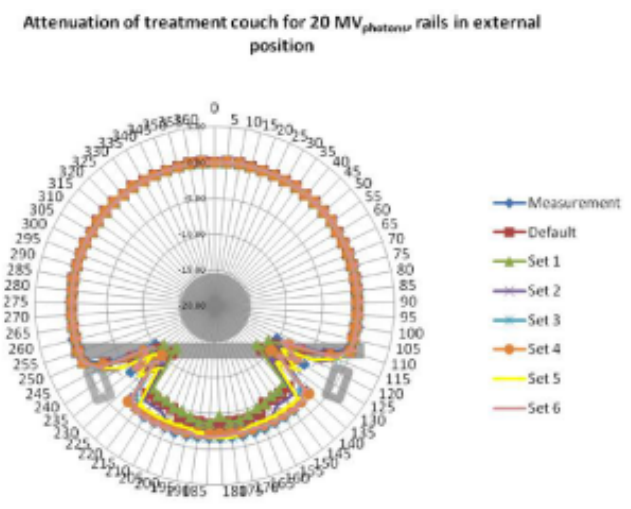

(d)

Figure 2: Attenuation of treatment couch. The measurements are shown in dark blue diamond, Set Default in dark red squares, Set 1 in green triangles, Set 2 in purple squares, Set 4 in orange circle, Set 5 in yellow stripes, and Set 6 in pink stripes. The grey shape indicates the measurement setup on treatment couch and the position of the rails.

a) Diagram of the attenuation of treatment couch for $6 \mathrm{MV}$

b) Diagram of the attenuation of treatment couch for $20 \mathrm{MV}_{\text {phons }}$, rails in middle position.

c) Diagram of the attenuation of treatment couch for $6 \mathrm{MV}_{\text {photons }}$, rails in external position.

d) Diagram of the attenuation of treatment couch for $20 \mathrm{MV}_{\text {photons }}$, rails in external position. 
and $-960 \mathrm{HU}$ for its filling. For the rails $\mathrm{HU}$ values of $100 \mathrm{HU}, 200 \mathrm{HU}$ and $250 \mathrm{HU}$ were set to find out the optimal modeling. The aberration between measured and calculated attenuation for Carbon plate and its filling for the Sets 2, 3 and 4 was still too high and therefore the $\mathrm{HU}$ values. For the rails an $\mathrm{HU}$ value of $100 \mathrm{HU}$ turned out to be too low, which could be verified by Set 5 . For the HU values of $200 \mathrm{HU}$ and $250 \mathrm{HU}$ for the rails good results for some measurements and a little bit too high aberrations for some measurements were found, respectively. In the end $225 \mathrm{HU}$ for the rails for Set 6 were taken and turned out to be optimal modeling. For the Carbon plate and its filling less $\mathrm{HU}$ values were taken for Set 5 and it was found that the HU values were too less. Therefore Set 6 was set to $-750 \mathrm{HU}$ and $-995 \mathrm{HU}$ for Carbon plate and its filling and turned out to be optimal modeling.

Carbon is a material which has low attenuation properties for the photon energies used in the radiotherapy (Meyer et al., 2001; Muthuswamy, 1999; Spezi et al., 2008). Therefore carbon is utilized for patient treatment couch and positioning devices of patients. Poppe et al. (2007); Myint et al. (2006) showed that the treatment couch is not neglectable during the calculation of dose distribution (Meara and Langmack, 1998; Myint et al., 2006; Poppe et al., 2007). In agreement with these statements we found in our analysis that the mean attenuation of Carbon plate was between $1.49 \%$ and $3.20 \%$ dependent on photon energy and gantry angle. In the region of rails the attenuation was higher. The rails are moveable and can mostly be moved out of the treatment field. This is possible for most 3D conformal treatment techniques with few treatment fields and gantry angles. For IMRT using seven to nine treatment fields and gantry angles, as well as for arc treatments or volumetric modulated arc treatment it is mostly not possible to move the rails out of treatment fields. For dynamic treatments it is necessary to consider the treatment couch during treatment planning to apply the correct dose to patient. The tumor control probability increases significantly if the uncertainty of applied dose can be minimized by $2 \%$. The attenuation of rails is $8.83 \%$ to $17.01 \%$ dependent on photon energy, position of rails underneath the treatment couch, gantry angle and therefore irradiated volume of the rails.

Mihaylov et al. (2008) showed in their study that contouring of treatment couch and attenuation information is necessary for the correct modeling of treatment couch in TPS to minimize the difference between calculated and applied dose (Mihaylov et al., 2008). Our results showed that the treatment couch can be correctly modeled in the TPS Eclipse. However, the default attenuation has to be corrected by typing in the correct $\mathrm{HU}$ values for different couch parts. One set for low and high photon energies is adequate for the correct modeling of treatment couch in TPS, just as described by Vanetti et al. (2009). In their study they analyzed the IGRT couch top from Varian Medical System for photon energies of $6 \mathrm{MV}_{\text {photons }}$ and $15 \mathrm{MV}_{\text {photons }}$. They showed that it is possible to verify the attenuation of treatment couch given from manufacturer as default using easy measurement setup. Due to our study we support this statement. However, it takes its time to find out the correct HU values and therefore the correct attenuation of treatment couch in TPS. Some sets of $\mathrm{HU}$ values for the different parts of treatment couch have to be tested. A fast way is to use a CT scan of treatment couch to verify the correct $\mathrm{HU}$ values of treatment couch.

McCormack et al. (2005) examined the magnitude of the effect over a range of posterior oblique gantry angles using a cylindrical solid water phantom containing an ionization chamber placed isocentrically. They found that a $6 \mathrm{MV}$ photon beam with a field size of $10 \times 5 \mathrm{~cm}$ was attenuated significantly as the gantry angle approached the plane of the couch from $2 \%$ at normal incidence and reaching $9 \%$ attenuation at angle of incidence 70 degrees. This could have serious implications regarding dose to the treatment volume for treatments requiring posterior oblique angles of incidence with a possible correction factor necessary in monitor unit calculations like breast cancer treatment and IMRT treatments with its many treatment fields and gantry angles. In our study the attenuation increased from $1.49 \%$ to $3.20 \%$ for Carbon table top and from $8.83 \%$ to $17.01 \%$ for rails dependent on photon energy and gantry angle.

The HU values for correct consideration of attenuation of treatment couch during treatment planning have to be typed in manually after inserting treatment couch in patient's CT dataset. There is no generalized possibility in administration to type in the correct $\mathrm{HU}$ values for each part of treatment couch. Due to the manually correction of $\mathrm{HU}$ values after inserting treatment couch can cause errors. Therefore the manufacturer is asked to provide a possibility for entering the verified $\mathrm{HU}$ values in administration.

\section{Conclusion}

The Varian Exact Couch can be modeled adequately in the TPS Eclipse. However, the HU values and therefore the attenuation have to be corrected manually for each patient. For Carbon plate an $\mathrm{HU}$ value of $-750 \mathrm{HU}$, for its filling of $-995 \mathrm{HU}$ and for the rails of $225 \mathrm{HU}$ have to be used for correct consideration of attenuation of treatment couch during treatment planning. For low and high photon energy one set of $\mathrm{HU}$ values can be used for correct modeling of treatment couch attenuation. The manufacturer is asked to provide a possibility for generalized entry of the verified $\mathrm{HU}$ values in administration to minimize the error source due to manually correction of HU values after inserting treatment couch to patients CT dataset.

\section{References}

1. Buckle $\mathrm{AH}$ (2005) An algorithm for detection of couch-beam intersection. Med Dosim 30: 65-68.

2. Butson MJ, Cheung T, Yu PK (2007) Megavoltage x-ray skin dose variation with an angle using grid carbon fibre couch tops. Phys Med Biol 52: 485-492.

3. De Ost B, Vanregemorter J, Schaeken B, Van den Weygaert D (1997) The effect of carbon fibre inserts on build-up and attenuation of high energy photon beams. Radiother Oncol 45: 272-277.

4. Galvin JM, Ezzell G, Eisbrauch A, Yu C, Butler B, et al. (2004) Implementing IMRT in clinical practice: a joint document of the American Society for Therapeutic Radiology and Oncology and the American Association of Physicists in Medicine. Int J Radiat Oncol Biol Phys 58: 1616-1634.

5. Gillis S, Bral S, De Wagter C, Derie C, Paelinck L, et al. (2005) Evaluation of the Sinmed Mastercouch as replacement for a standard couch. Radiother Oncol 75: 227-236.

6. Higgins DM, Whitehurst P, Morgan AM (2001) The effect of carbon fiber couch inserts on surface dose with beam size variations. Med Dosim 26: 251-254.

7. McCormack S, Diffey J, Morgan A (2005) The effect of gantry angle on megavoltage photon beam attenuation by a carbon fiber couch insert. Med Phys 32: 483-487.

8. Meara SJ, Langmack KA (1998) An investigation into the use of carbon fibre for megavoltage radiotherapy applications. Phys Med Biol 43: 1359-1366.

9. Meydanci TP, Kemikler G (2008) Effect of a carbon fiber tabletop on the surface dose and attenuation for high-energy photon beams. Radiat Med 26: 539-544.

10. Meyer J, Mills JA, Haas OC, Burnham KJ, Parvin EM (2001) Accommodation of couch constraints for coplanar intensity modulated radiation therapy. Radiother Oncol 61: 23-32.

11. Mihaylov IB, Corry P, Yan Y, Ratanatharathorn V, Moros EG (2008) Modeling of carbon fiber couch attenuation properties with a commercial treatment planning system. Med Phys 35: 4982-4988. 
Citation: Wagner D, Vorwerk H (2011) Treatment Couch Modeling in the Treatment Planning System Eclipse. J Cancer Sci Ther 2: 007-012. doi:10.4172/1948-5956.1000049

12. Munjal RK, Negi PS, Babu AG, Sinha SN, Anand AK, et al. (2006) Impact of $6 \mathrm{MV}$ photon beam attenuation by carbon fiber couch and immobilization divices in IMRT planning and dose delivery. J Med Phys 31: 67-71.

13. Muthuswamy MS (1999) A method of beam couch intersection detection. Med Phys 26: 229-235.

14. Myint WK, Niedbala M, Wilkins D, Gerig LH (2006) Investigating treatment dose error due to beam attenuation by carbon fiber tabletop. J Appl Clin Med Phys 7: 21-27.

15. Poppe B, Chofor N, Rühmann A, Kunth W, Djouguela A, et al. (2007) The effect of a carbon.fiber couch on the depth-dose curves and transmission properties for megavoltage photon beams. Strahlenther Onkol 183: 43-48.

16. Spezi E, Angelini AL, Romani F, Guido A, Bunkheila F, et al. (2008) Evaluating the influence of the Siemens IGRT carbin fibre tabletop in head and neck IMRT. Radiother Onkol 89: 114-122.
17. Spezi E, Ferri A (2007) Dosimetric characteristics of Siemens IGRT carbon fiber tabletop. Med Dosim 32: 295-298.

18. Ulmer W, Pyyry J, KaissI W (1995) A Triple Gaussian Pencil Beam Model for Photon Beam Treatment Planning Phys Med Biol 50: 1767-1790.

19. Ulmer W, Pyyry J, KaissI W (2005) A 3D photon superposition/convolution algorithm and its foundation on results of Monte Carlo calculations Phys Med Biol 50: 1767-1790.

20. Vanetti E, Nicolini G, Clivio A, Fogliata A, Cozzi L (2009) The impact of treatment couch modelling on RapidArc. Phys Med Biol 54: N157-N166.

21. Vieira SC, Kaatee RS, Dirkx ML, Heijmen BJ (2003) Two-dimensiona measurement of photon beam attenuation by the treatment couch and immobilization devices using an electronic portal imaging device. Med Phys 30: $2981-2987$ 